\title{
ADT in the diagnosis of atopic dermatitis - preliminary tests
}

by M. Kaczmarek*

\author{
* Gdansk Univ. of Technology, 80-233, Narutowicza Str., Gdansk, Poland, mariusz.kaczmarek@eti.pg.edu.pl
}

\begin{abstract}
The article describes the proposal to use Active Dynamic Thermography in the diagnosis and monitoring of the progress of atopic dermatitis treatment. Preliminary studies were performed on a group of 20 volunteers: 10 people affected at various stages and 10 healthy as a reference. The study consisted of recording a sequence of static thermograms, followed by cooling the surface with a fan and recording the thermograms during and after cooling. The results were compared in a group of patients with atopic dermatitis with a group of healthy people.
\end{abstract}

\section{Introduction}

Atopic dermatitis is a common, chronic and recurrent inflammatory skin disease. Typical symptoms of this disease are persistent itching and dry skin. Changes can occur on the entire body surface, but most often they are located on the back, thighs, elbows, also on the face. Various methods of description are used to assess its severity and stage. Unfortunately, these are not objective methods. They are based on subjective assessment by a doctor. The most popular methods of evaluating the state of the disease include: Scoring Atopic Dermatitis Index (SCORAD) and Eczema Area and Severity Index (EASI) are best known. Other methods for measuring the severity of atopic dermatitis include: Investigators' Global Assessment (IGA), Patient-oriented Eczema Measure (POEM), Six Area, Six Sign Atopic Dermatitis (SASSAD), Three Item Severity (TIS), Simple Scoring System (SSS), Basic Clinical Scoring System (BCSS), PatientOriented Scoring of Atopic Dermatitis (PO-SCORAD). Of all the available tools to assess the severity of atopic dermatitis, SCORAD is the most widely used scale in the world. A lot of research has been done to validate this scale and it has been found to be a reliable tool in both clinical trials and everyday clinical practice $[1,2,3]$. The maximum score on this scale is 103 points, with $60 \%$ of the total score being the severity of the lesions, and $20 \%$ by extent and subjective symptoms [11, 12]. Due to the obtained result, atopic dermatitis is classified as mild ( $<25$ points), moderate (25-50 points) and severe (> 50 points).

IGA is a general assessment of the severity of atopic dermatitis. The most commonly used scale is 6 ( 0 - no changes, 1 - almost no changes, 2 - mild severity, 3 - moderate severity, 4 - high severity, 5 - very high severity).

An effective, intensive treatment for severe atopic dermatitis involves wrapping the affected area with topical corticosteroids and wet bandages. Alternative therapy is light therapy (phototherapy) involves exposing the skin to controlled amounts of natural sunlight. Other forms use artificial ultraviolet A (UVA) and narrow band ultraviolet $B$ (UVB) either alone or with medications. It is important to know if the therapy is effective and if the patient's condition is improving, or if another form of treatment should be used.

The Active Dynamic Thermography (ADT) method [4] was used to find an objective method to assess the severity of atopic dermatitis and evaluate treatment progress. Technologies which are the subject of the work, called active dynamic thermography significantly differ from known thermal infrared imaging - static thermography. The basic tool for measuring the ADT remains an infrared camera, which is used to record time-varying temperature distributions, but a necessary complement to the apparatus generating an external thermal excitation (heating or cooling of the tissue). The principle of operation of diagnostic system is not based on the determination of the temperature but thermal properties of the tissue, which greatly measurement becomes independent of external/ambient conditions. An essential element differs these two techniques is the use of external thermal stimulation and analysis of transient data using a mathematical thermal model of the test object. Transient processes that depend on the thermal properties of the tissues are tested. It presents the results of the analysis as substitute parametric images, such as thermal time constants, which are strongly correlated with the physiological properties of the examined tissue. Developed and optimized systems ADT using such imaging and developed software is now suitable for implementation into clinical practice [5].

\section{Method and Materials}

Preliminary studies were performed on a group of 20 volunteers: 10 people affected at various stages of atopic dermatitis disease (ADD) and 10 healthy as a reference. Patients were evaluated by the physician according to the SCORAD and IGA scales. The mean SCORAD index was $27.89 \pm 15$ and IGA index was $1.9 \pm 0.87$, next the patients was examined by ADT method. The study consisted of registering a sequence of static thermograms, followed by cooling the surface with a fan (lasting 40s) and recording the thermograms during and after cooling (120s). The A655SC thermal camera was used with spatial resolution of $640 \times 480$ pixels and thermal resolution of $30 \mathrm{mK}$. 
Based on the recorded thermal sequences, parametric images and scores was calculated: thermal time constants (fitting to exponential model $-\tau_{1}, \tau_{2}$ ) and TSR coefficients. The results obtained were compared within the group (sick, healthy) and between groups.

\section{Results}

In the article we show the preliminary results of the experiment with patients. The method definitely requires optimization, even in the selection of external stimulation. In the described study a fan was used as a cooling stimulus, i.e. a relatively weak stimulus. The skin surface was cooled by 2 degrees Celsius. Further studies are planned with patients with different stages of the disease and at different ages. The figures below show examples of recorded thermograms for two patient cases along with a simple analysis - temperature difference 60 seconds after the cooling stimulus stops. It is clearly visible that areas with atopic dermatitis heat up faster after cooling - the yellow and red areas.
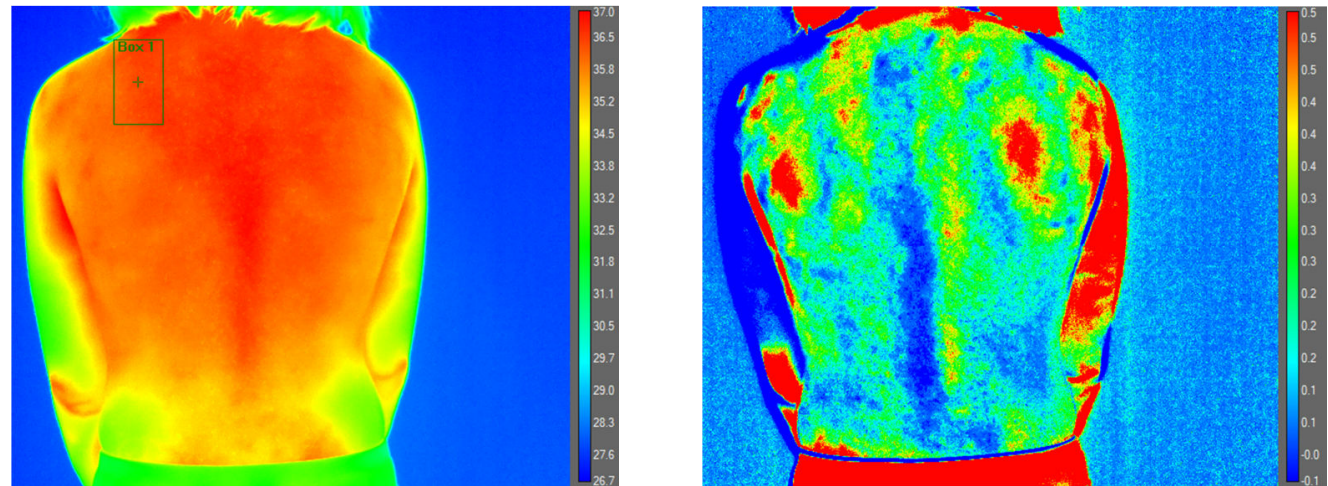

Fig. 1. A patient with atopic dermatitis on the back scored as SCORAD=18 and $I G A=1$ with $R O I$ for simple analysis

Analysis of thermographic sequences requires preprocessing. Before determining parametric images, thermograms must be aligned (registered), because the test takes several minutes and it is possible to stand still for such a long time. Changes in the skin are often punctual, so when determining the region of interest you must remember that it covers the skin with varying degrees of severity of skin changes. Therefore, the result should be analysed as the distribution of a certain structure (texture, pattern) and not a uniform surface. As a result of the analysis, a correlation was found between the value of variance and standard deviation for the region and the skin condition, and not the parameter value itself.

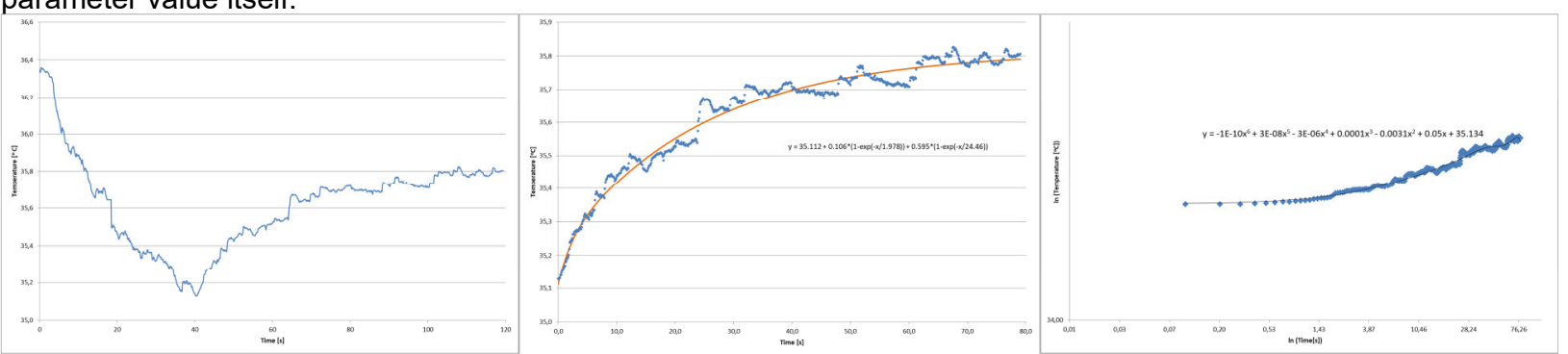

Fig. 2. Simple results for Patient from Fig.1: Temperature vs Time; exponential fitting with time constants, TSR fitting

Hair placement on the skin surface is another factor impeding the interpretation of results. Hair can be treated as skin changes. We are now investigating the effect of using morphological filtration to remove these artefacts (hair) on the acquired parameters of skin lesions.

\section{REFERENCES}

[1] Schmitt J., Langan S., Deckert S., Svensson A., Kobyletzki L., Assessment of clinical signs of atopic dermatitis: a systematic review and recommendation. J Allergy Clin Immunol 2013, 132, 1337-1347, 2013.

[2] Rehal B., Armstrong A.W.: Health outcome measures in atopic dermatitis: a systematic review of trends in disease severity and quality-of-life instruments 1985-2010. PLoS One 2011, 6, e17520, 2010.

[3] Severity scoring of atopic dermatitis: the SCORAD index. Consensus report of the European Task Force on Atopic Dermatitis. Dermatology 1993, 186, 23-31, 1993.

[4] Kaczmarek M., Nowakowski A., Active IR-Thermal Imaging in Medicine, J Nondestruct Eval (2016), Springer, 35:19, DOI 10.1007/s10921-016-0335-y, 2016.

[5] Renkielska A., Nowakowski A., Kaczmarek M., et al., Burn depths evaluation based on active dynamic IR thermal imaging - a preliminary study. Burns; 32: pp. 867-875, 2006. 